\title{
Mode Dispersion and Delay Characteristics of Optical Waveguides Using Equivalent TL Circuits
}

\author{
Anthony C. Boucouvalas, Fellow, IEEE and Xin Qian, Student Member, IEEE
}

\begin{abstract}
A new analysis leading to an exact and efficient algorithm is presented for calculating directly and without numerical differentiation the mode dispersion characteristics of cylindrical dielectric waveguides of arbitrary refractive-index profile. The new algorithm is based on the equivalent transmission-line (T-L) technique. From Maxwell's equations, we derive an equivalent $T$ - $L$ circuit for a cylindrical dielectric waveguide. Based on the TL-circuit model we derive exact analytic formulas for a recursive algorithm which allows direct calculation of mode delay and dispersion. We demonstrate this technique by calculating the fundamental mode dispersion for step, triangular, and linear chirp optical fiber refractive index profiles. The accuracy of the numerical results is also examined. The proposed algorithm computes dispersion directly from the propagation constant without the need for curve fitting and subsequent successive numerical differentiation. It is exact, rapidly convergent, and it results in savings for both storage memory and computing time.
\end{abstract}

Index Terms-Fiber-optic mode dispersion, optical communications, optical waveguides, transmission-line (T-L) techniques.

\section{INTRODUCTION}

C ALCULATION of waveguide mode propagation constants as a function of wavelength for optical fibers is a well-established problem and many different solution methods have been proposed, studied, and implemented. For long distance high capacity transmission applications, an important metric of optical fibers is pulse dispersion in picoseconds per nanometers per kilometers. Understanding and controlling the variation of dispersion against wavelength is essential for the design of optical fiber systems and fibers with more sophisticated refractive-index profiles, and of more suitable dispersion characteristics such as dispersion-shifted and dispersion flattened fibers have been extensively studied and installed in the field [1]. Numerical techniques for fast calculation of total mode dispersion from the mode propagation constant ideally should be as direct as possible. The methods must be theoretically exact hence correct prediction of even small values of mode dispersion would be possible and they must allow for the inclusion of material dispersion component. Analytical direct techniques are preferred in order to avoid high order curve fitting and subsequent numerical differentiation of data.

The definition of dispersion involves the use of first (delay) and second (dispersion) derivatives of mode propagation constant with respect to wavelength, thus theoretical evaluation

Manuscript received February 2, 2005; revised March 23, 2005.

The authors are with the Microelectronics and Multimedia Research Center, Bournemouth University, Dorset BH12 5BB, U.K. (e-mail: tboucouv@bournemouth.ac.uk; qxin@bournemouth.ac.uk).

Digital Object Identifier 10.1109/JQE.2005.848918 of dispersion requires the determination of such derivatives in the first instance. However, direct numerical calculation of the first and second derivatives from data points of mode propagation constants versus wavelength based on simple finite differences can result in errors due to approximations [2]. Different improved procedures have then been proposed, aiming at obtaining good accuracy in calculation of the dispersion coefficient [3]-[5]. Mammel and Cohen [3] proposed the Rayleigh quotient to obtain the first derivative of the propagation constant, but they used direct numerical differentiation in the calculation of the second derivative. E. K. Sharma et al. [4] avoided numerical differentiations by solving three differential equations for the propagation constant and its first and second derivatives, respectively. Recently, A. Sharma and Banerjee [5] reported another method based on a matrix perturbation theory and showed that computational effort can be reduced compared to the method of E. K. Sharma et al. [4].

We have shown that equivalent transmission-line (T-L) circuit techniques are most powerful and can be easily applied to optical fibers in order to determine exactly the mode propagation constants [6].

In this paper we extend the theory and present a novel method based on the T-L circuit technique for calculating the dispersion of optical fibers of known but arbitrary refractiveindex profiles. First, we derive the equations for the derivatives of the propagation constant with respect to the wavelength analytically. By using a recursive formula, we show that for a given wavelength, the first derivative can be expressed in terms of equivalent circuit impedances at the wavelength of interest and the second derivative can be expressed in terms of circuit impedances and the first derivative. Second, by calculating the derived equivalent circuit formulas, we are able to work out the impedances at the specified wavelength using the T-L technique. Once these along with the material dispersion information are given, the total dispersion can be accurately calculated from its definition. Numerical results on convergence speed for our method as compared with the methods [1], [7], [8] will be given. The following section describes the basic theory our technique is based upon.

\section{TRANSMISSION LINE THEORY}

Our modeling divides a cylindrical symmetric optical fiber into a large number of concentric homogeneous cylindrical layers of thickness $\delta r$, permittivity $\varepsilon$, permeability $\mu$, and conductivity $\sigma$ in Fig. 1. 


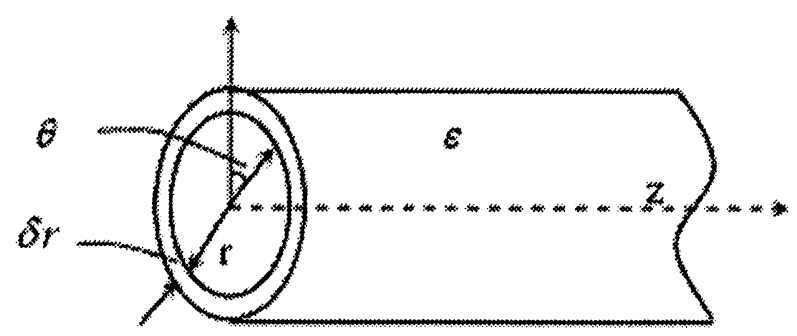

Fig. 1. Homogeneous optical fiber thin cylindrical layer.

Using Maxwell's equations for the $E$ and $H$ fields we drive the following equations for any such layer [9]

$$
\begin{aligned}
& \left.\begin{array}{l}
\beta r E_{\theta}-l E_{Z}=\omega \mu r H_{r} \\
l H_{Z}-\beta r H_{\theta}=(\omega \varepsilon-j \sigma) r E_{r} \\
\frac{\partial\left(\omega \mu r H_{r}\right)}{\partial r}=-j \omega \mu\left(l H_{\theta}+\beta r H_{Z}\right)
\end{array}\right\} \\
& \frac{\partial\left[(\omega \varepsilon-j \sigma) r E_{r}\right]}{\partial r}=-(\sigma+j \omega \varepsilon)\left(l E_{\theta}+\beta r E_{Z}\right) \\
& \frac{\partial\left(l H_{\theta}+\beta r H_{Z}\right)}{\partial r}=-\frac{\gamma^{2}}{j \omega \mu} \omega \mu r H_{r}+\beta H_{Z}-\frac{l}{r} H_{\theta} \\
& \frac{\partial\left(l E_{\theta}+\beta r E_{Z}\right)}{\partial r}=-\frac{\gamma^{2}}{\sigma+j \omega \varepsilon}(\omega \varepsilon-j \sigma) r E_{r} \\
& +\beta \mathrm{E}_{Z}-\frac{l}{r} E_{\theta}
\end{aligned}
$$

where $\gamma^{2}=\beta^{2}+(l / r)^{2}-\omega^{2} \mu \varepsilon+j \omega \mu \sigma, \beta$ is the propagation constant, $l$ is the azimuthal mode number (integer), and $\omega$ is the mode frequency. For the case where $\sigma=0, \mu=\mu_{0}, \varepsilon=n^{2} \varepsilon_{0}$, with $n$, the refractive index of the layer at distance $r$ from the axis.

After some algebra similarly to [9], (1) and (2) can be transformed into

$$
\left.\begin{array}{l}
\frac{\partial V_{s}}{\partial r}=\frac{-\gamma_{s}^{2}}{j \omega \varepsilon_{0} n F} I_{s} \\
\frac{\partial I_{s}}{\partial r}=-j \omega \varepsilon_{0} n F V_{s}
\end{array}\right\}
$$

where $\gamma_{d^{s}}^{2}=\beta^{2}+((l / r))^{2}-n^{2} k_{0}^{2} \mp\left(2 n k_{0} \beta l\right) /\left((\beta r)^{2}+l^{2}\right)(-$ for $H E,+$ for $E H$ modes), $F=\left((\beta r)^{2}+l^{2}\right) /(r)$. Equations (3) and (4) represent two independent transmission lines with voltages $V_{s}, V_{d}$ and currents $I_{s}, I_{d}$. The corresponding characteristic impedances are

$$
\left.\begin{array}{l}
Z_{s}=\frac{\gamma_{s}}{j \omega \varepsilon_{0} n F} \\
Z_{d}=\frac{\gamma_{d}}{j \omega \varepsilon_{0} n F}
\end{array}\right\}
$$

Equations (3) and (4) are recognized as T-L equations the solution of which can be represented by the following equivalent electric circuit, Fig. 2.

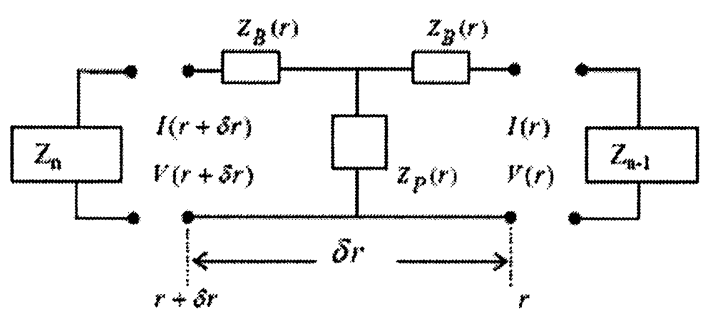

Fig. 2. Equivalent circuit of a dielectric waveguide layer.

The transmission line impedances are given by

$$
\left.\begin{array}{l}
Z_{B}=Z_{d^{s}} \tanh \left(\gamma_{d^{s}} \frac{\delta r}{2}\right) \\
Z_{P}=\frac{Z_{d^{s}}}{\sinh \left(\gamma_{d^{s}} \delta r\right)}
\end{array}\right\}
$$

where $\delta r$ is the length of the transmission line (the thickness of cylindrical layers)

$$
\left.\begin{array}{l}
Z_{B}=\sinh \left(\gamma_{d^{s}} \delta r\right) \tanh \left(\gamma_{d^{s}} \frac{\delta r}{2}\right) Z_{P} \\
Z_{P}=\frac{\gamma_{d^{s}} Z_{0}}{j n r k_{0}\left(\beta^{2}+\left(\frac{l}{r}\right)^{2}\right) \sinh \left(\gamma_{d^{s}} \delta r\right)}
\end{array}\right\} .
$$

Since $\delta r$ is infinitesimal, $(\delta r) /(r) \ll 1$, from (7) we can have

$$
\left.\begin{array}{l}
Z_{B}=\frac{1}{2}(\delta r)^{2} \gamma_{d^{s}}^{2} Z_{P} \\
Z_{P}=\frac{Z_{0}}{j n r \delta r k_{0}\left(\beta^{2}+\left(\frac{l}{r}\right)^{2}\right)}
\end{array}\right\} .
$$

An optical fiber can be represented as a cascade of TL circuits connected in tandem. The mode propagation constants can be determined when the optical energy is trapped inside the optical waveguide, and this is equivalent to the resonance conditions $\left(Z_{\text {total }}=0\right)$ of the equivalent TL circuits [10]. The series is terminated with the characteristic impedance of the medium at the axis $(r=0)$ of the fiber, and the characteristic impedance of the outer cladding $(r=\infty)$. We can find $Z_{\text {in }}$ the total impedance from $r=0$ up to the core-cladding boundary and similarly, $Z_{\text {out }}$ the total impedance to that boundary by using circuit theory starting from large $r(r=\infty)$ in the cladding

$$
\begin{aligned}
Z_{\substack{\text { in } \\
\text { out }}}=Z_{B}(a \pm 1) & 1 \\
& +\frac{1}{\frac{1}{Z_{P}(a \pm 1)}+\frac{1}{Z_{B}(a \pm 1)+Z_{B}(a \pm 2)+\frac{1}{: !}}}
\end{aligned}
$$

where $Z_{\text {prev }}$ is the characteristic impedance at $r=\infty$ when the positive sign is used or it becomes the characteristic impedance at $r=0$ when the negative sign is used, $a$ is the core-cladding interface radius. The total circuit resonates when $Z_{\text {in }}$ and $Z_{\text {out }}$ are equal and opposite, hence $Z_{\text {total }}=Z_{\text {in }}+Z_{\text {out }}=0$, at the propagation constant of any mode. Following this technique, we can obtain the unknown mode propagation constant $\beta$ using the root searching method which locates the roots of the total impedance of the TL circuits. 
The equivalent TL circuit impedances (7) or (8) are functions of wavelength and the propagation constant, so the first and second derivatives of the propagation constant can be extended as follows:

$$
\begin{aligned}
\frac{\partial \beta}{\partial \lambda} & =\frac{\left.\frac{\partial Z_{N}}{\partial \lambda}\right|_{\beta=\beta_{0}}}{\left.\frac{\partial Z_{N}}{\partial \beta}\right|_{\lambda=\lambda_{0}}} \\
\frac{\partial^{2} \beta}{\partial \lambda^{2}} & =\frac{\partial}{\partial \lambda}\left[\frac{\partial \beta}{\partial \lambda}\right]=\frac{\frac{\partial^{2} Z_{N}}{\partial \lambda^{2}}-\frac{\partial \beta}{\partial \lambda} \frac{\partial^{2} Z_{N}}{\partial \beta \partial \lambda}}{\frac{\partial Z_{N}}{\partial \beta}}
\end{aligned}
$$

where $\beta_{0}$ is the propagation constant at the wavelength of interest $\lambda_{0}$ [10]. $Z_{N}$ can be calculated recurrently from $Z_{n}=$ $Z_{B}+\left((1) /\left(Z_{B}+Z_{n-1}\right)+(1) /\left(Z_{P}\right)\right)^{-1},(n=1,2 \ldots N)$, which is the $n$th characteristic impedance of cylindrical layers. $\mathrm{N}$ is the total number of cylindrical layers.

Equations (10) and (11) are equations related to derivatives of the impedances in each transmission line. The recursive equations allow us to determine delay and dispersion directly from equivalent TL circuit characteristic impedances.

\section{SOLUTION PROCEDURE}

It is well known that the total dispersion in the single-mode regime is composed of two components, material and waveguide dispersion. The concept of zero total dispersion by cancellation of the material and waveguide dispersions was proposed as long ago as 1970 by Dyott and Stern [11]. The waveguide dispersion arises from the variation in group velocity. It depends not only on the core radius and the refractive index difference between the core and the cladding of optical fibers, but also on the shape of the refractive index profile. The material contribution results from the wavelength dependence of the refractive index. Our algorithm allows calculation of both material and waveguide dispersions. The material refractive index dependence on wavelength [8] is included in our calculations and it is given by:

$$
\begin{aligned}
n_{1}(\lambda)=C_{0}+C_{1} \lambda^{2} & +C_{2} \lambda^{4}+\frac{C_{3}}{\lambda^{2}-0.035} \\
& +\frac{C_{4}}{\left(\lambda^{2}-0.035\right)^{2}}+\frac{C_{5}}{\left(\lambda^{2}-0.035\right)^{3}}
\end{aligned}
$$

where $C_{0}=1.4508554, C_{1}=-0.0031268, C_{2}=$ $-0.0000381, C_{3}=0.0030270, C_{4}=-0.0000779, C_{5}=$ 0.0000018 .

The following steps detail our solution procedure for the mode delay and dispersion.

From (10) and (11), delay and dispersion equations are given by [12]

$$
\begin{aligned}
\tau & =\frac{L \lambda^{2}}{2 \pi c} \frac{\partial \beta}{\partial \lambda} \\
D & =\frac{1}{L} \frac{\partial \tau}{\partial \lambda}=\frac{1}{2 \pi c}\left(2 \lambda \frac{\partial \beta}{\partial \lambda}+\lambda^{2} \frac{\partial^{2} \beta}{\partial \lambda^{2}}\right)
\end{aligned}
$$

where $\tau$ is the delay, $D$ is the dispersion, $L$ is the optical fiber length, and $c$ is the velocity of light in free space.
We next derive equations for $\left(\partial Z_{N}\right) /(\partial \lambda),\left(\partial Z_{N}\right) /(\partial \beta),\left(\partial^{2} Z_{N}\right) /\left(\partial \lambda^{2}\right),\left(\partial^{2} Z_{N}\right) /(\partial \beta \partial \lambda)$ analytically which are to be used in the recursive algorithm

$$
\begin{aligned}
& \frac{\partial Z_{N}}{\partial \lambda}=\frac{\partial Z_{B}}{\partial \lambda}+\left(\frac{1}{Z_{B}+Z_{n-1}}+\frac{1}{Z_{P}}\right)^{-2} \\
& \times\left(\frac{\frac{\partial Z_{B}}{\partial \lambda}+\frac{\partial Z_{n-1}}{\partial \lambda}}{\left(Z_{B}+Z_{n-1}\right)^{2}}+\frac{\frac{\partial Z_{P}}{\partial \lambda}}{Z_{P}^{2}}\right) \\
& \left.\frac{\partial Z_{N}}{\partial \beta}=\frac{\partial Z_{B}}{\partial \beta}+\left(\frac{1}{Z_{B}+Z_{n-1}}+\frac{1}{Z_{P}}\right)^{-2}\right\} \\
& \times\left(\frac{\frac{\partial Z_{B}}{\partial \beta}+\frac{\partial Z_{n-1}}{\partial \beta}}{\left(Z_{B}+Z_{n-1}\right)^{2}}+\frac{\frac{\partial Z_{P}}{\partial \beta}}{Z_{P}^{2}}\right) \\
& \frac{\partial^{2} Z_{N}}{\partial \lambda^{2}}=\frac{\partial^{2} Z_{B}}{\partial \lambda^{2}}+2\left(\frac{1}{Z_{B}+Z_{n-1}}+\frac{1}{Z_{P}}\right)^{-3} \\
& \times\left(\frac{\frac{\partial Z_{B}}{\partial \lambda}+\frac{\partial Z_{n-1}}{\partial \lambda}}{\left(Z_{B}+Z_{n-1}\right)^{2}}+\frac{\frac{\partial Z_{P}}{\partial \lambda}}{Z_{P}^{2}}\right)^{2} \\
& +\left(\frac{1}{Z_{B}+Z_{n-1}}+\frac{1}{Z_{P}}\right)^{-2}\left(\frac{\frac{\partial^{2} Z_{B}}{\partial \lambda^{2}}+\frac{\partial^{2} Z_{n-1}}{\partial \lambda^{2}}}{\left(Z_{B}+Z_{n-1}\right)^{2}}\right. \\
& \left.-\frac{2\left(\frac{\partial Z_{B}}{\partial \lambda}+\frac{\partial Z_{n-1}}{\partial \lambda}\right)^{2}}{\left(Z_{B}+Z_{n-1}\right)^{3}}+\frac{\frac{\partial^{2} Z_{P}}{\partial \lambda^{2}}}{Z_{P}^{2}}-\frac{2\left(\frac{\partial Z_{P}}{\partial \lambda}\right)^{2}}{Z_{P}^{3}}\right) \\
& \frac{\partial^{2} Z_{N}}{\partial \beta \partial \lambda}=\frac{\partial^{2} Z_{B}}{\partial \beta \partial \lambda}+2\left(\frac{1}{Z_{B}+Z_{n-1}}+\frac{1}{Z_{P}}\right)^{-3} \\
& \times\left(\frac{\frac{\partial Z_{B}}{\partial \lambda}+\frac{\partial Z_{n-1}}{\partial \lambda}}{\left(Z_{B}+Z_{n-1}\right)^{2}}+\frac{\frac{\partial Z_{P}}{\partial \lambda}}{Z_{P}^{2}}\right) \\
& \times\left(\frac{\frac{\partial Z_{B}}{\partial \beta}+\frac{\partial Z_{n-1}}{\partial \beta}}{\left(Z_{B}+Z_{n-1}\right)^{2}}+\frac{\frac{\partial Z_{P}}{\partial \beta}}{Z_{P}^{2}}\right) \\
& +\left(\frac{1}{Z_{B}+Z_{n-1}}+\frac{1}{Z_{P}}\right)^{-2}\left(\frac{\frac{\partial^{2} Z_{B}}{\partial \beta \partial \lambda}+\frac{\partial^{2} Z_{n-1}}{\partial \beta \partial \lambda}}{\left(Z_{B}+Z_{n-1}\right)^{2}}\right. \\
& -\frac{2\left(\frac{\partial Z_{B}}{\partial \lambda}+\frac{\partial Z_{n-1}}{\partial \lambda}\right)\left(\frac{\partial Z_{B}}{\partial \beta}+\frac{\partial Z_{n-1}}{\partial \beta}\right)}{\left(Z_{B}+Z_{n-1}\right)^{3}} \\
& \left.+\frac{\frac{\partial^{2} Z_{P}}{\partial \beta \partial \lambda}}{Z_{P}^{2}}-\frac{2 \frac{\partial Z_{P}}{\partial \lambda} \frac{\partial Z_{P}}{\partial \beta}}{Z_{P}^{3}}\right)
\end{aligned}
$$

The first derivatives of the impedance equations (8) as a function of the fiber optic layer physical and optical parameters are given by

$$
\left.\begin{array}{rl}
\frac{\partial Z_{P}}{\partial \beta} & =\frac{-2 Z_{0} \beta}{n r \delta r k_{0}\left(\beta^{2}+\left(\frac{l}{r}\right)^{2}\right)^{2}} \\
\frac{\partial Z_{P}}{\partial \lambda} & =\frac{-Z_{0}}{r \delta r k_{0}\left(\beta^{2}+\left(\frac{l}{r}\right)^{2}\right)^{2}} \frac{\frac{\partial n}{\partial \lambda}}{n^{2}}
\end{array}\right\}
$$


where

$$
\begin{aligned}
\gamma^{2}= & \left.\beta^{2}+\left(\frac{l}{r}\right)^{2}-n^{2} k_{0}^{2}-\frac{2 n \beta l k_{0}}{(\beta r)^{2}+l^{2}}\right) \\
\frac{\partial \gamma^{2}}{\partial \beta}= & 2 \beta-\frac{2 n l k_{0}\left(l^{2}-(\beta r)^{2}\right)}{\left((\beta r)^{2}+l^{2}\right)^{2}} \\
\frac{\partial \gamma^{2}}{\partial \lambda}= & -2 n \frac{\partial n}{\partial \lambda} k_{0}^{2}-\frac{2 k_{0} \beta l}{(\beta r)^{2}+l^{2}} \frac{\partial n}{\partial \lambda} \\
\frac{\partial n}{\partial \lambda}= & 2 C_{1} \lambda+4 C_{2} \lambda^{3}-\frac{2 C_{3} \lambda}{\left(\lambda^{2}-0.035\right)^{2}} \\
& -\frac{4 \mathrm{C}_{4} \lambda}{\left(\lambda^{2}-0.035\right)^{3}}-\frac{6 C_{5} \lambda}{\left(\lambda^{2}-0.035\right)^{4}} .
\end{aligned}
$$

For the second derivatives of the impedance equations (8)

$$
\begin{aligned}
& \frac{\partial^{2} Z_{P}}{\partial \beta \partial \lambda}=\frac{2 Z_{0} \beta}{r \delta r k_{0}\left(\beta^{2}+\left(\frac{l}{r}\right)^{2}\right)^{2}} \frac{\frac{\partial n}{\partial \lambda}}{n^{2}} \\
& \left.\frac{\partial^{2} Z_{P}}{\partial \lambda^{2}}=\frac{-Z_{0}}{r \delta r k_{0}\left(\beta^{2}+\left(\frac{l}{r}\right)^{2}\right)}\left(\frac{\frac{\partial^{2} n}{\partial \lambda^{2}}}{n^{2}}-\frac{2\left(\frac{\partial n}{\partial \lambda}\right)^{2}}{n^{3}}\right)\right\} \\
& \frac{\partial^{2} Z_{B}}{\partial \beta \partial \lambda}=\frac{\delta r^{2}}{2}\left(\frac{\partial^{2} \gamma^{2}}{\partial \beta \partial \lambda} Z_{P}+\frac{\partial \gamma^{2}}{\partial \beta} \frac{\partial Z_{P}}{\partial \lambda}+\frac{\partial \gamma^{2}}{\partial \lambda} \frac{\partial Z_{P}}{\partial \beta}+\gamma^{2} \frac{\partial^{2} Z_{P}}{\partial \beta \partial \lambda}\right) \\
& \frac{\partial^{2} Z_{B}}{\partial \lambda^{2}}=\frac{\delta r^{2}}{2}\left(\frac{\partial^{2} \gamma^{2}}{\partial \lambda^{2}} Z_{P}+2 \frac{\partial \gamma^{2}}{\partial \lambda} \frac{\partial Z_{P}}{\partial \lambda}+\gamma^{2} \frac{\partial^{2} Z_{P}}{\partial \lambda^{2}}\right)
\end{aligned}
$$

where

$$
\begin{aligned}
\frac{\partial^{2} \gamma^{2}}{\partial \beta \partial \lambda}= & \frac{-2 l k_{0}\left(l^{2}-(\beta r)^{2}\right)}{\left((\beta r)^{2}+l^{2}\right)^{2}} \frac{\partial n}{\partial \lambda} \\
\frac{\partial^{2} \gamma^{2}}{\partial \lambda^{2}}= & -2 k_{0}^{2}\left(\left(\frac{\partial n}{\partial \lambda}\right)^{2}+n \frac{\partial^{2} n}{\partial \lambda^{2}}\right)-\frac{2 \beta k_{0} l}{(\beta r)^{2}+l^{2}} \frac{\partial^{2} n}{\partial \lambda^{2}} \\
\frac{\partial^{2} n}{\partial \lambda^{2}}= & 2 C_{1}+12 C_{2} \lambda^{2}+\frac{6 C_{3} \lambda^{2}+0.07 C_{3}}{\left(\lambda^{2}-0.035\right)^{3}} \\
& +\frac{20 C_{4} \lambda^{2}+0.14 C_{4}}{\left(\lambda^{2}-0.035\right)^{4}}-\frac{42 C_{5} \lambda^{2}+0.21 C_{5}}{\left(\lambda^{2}-0.035\right)^{5}}
\end{aligned}
$$

To test the accuracy of (17)-(20) derived from the approximate impedance equations (8), we have also worked out the exact first and second derivatives from the exact impedance equations (7), in Appendix A. We have found that (17)-(20) are accurate for our purposes. Approximations are not however essential in this analysis and if the exact equivalent equations are preferred they can be used and we have included them in Appendix A. In this solution procedure, almost all the computation time is spent in calculating (15) and (16). The derivatives $\left(\partial Z_{N}\right) /(\partial \lambda),\left(\partial Z_{N}\right) /(\partial \beta)$ and $\left(\partial^{2} Z_{N}\right) /\left(\partial \lambda^{2}\right),\left(\partial^{2} Z_{N}\right) /(\partial \beta \partial \lambda)$ can now be obtained very efficiently. Therefore, delay and dispersion can be calculated accurately and recursively.

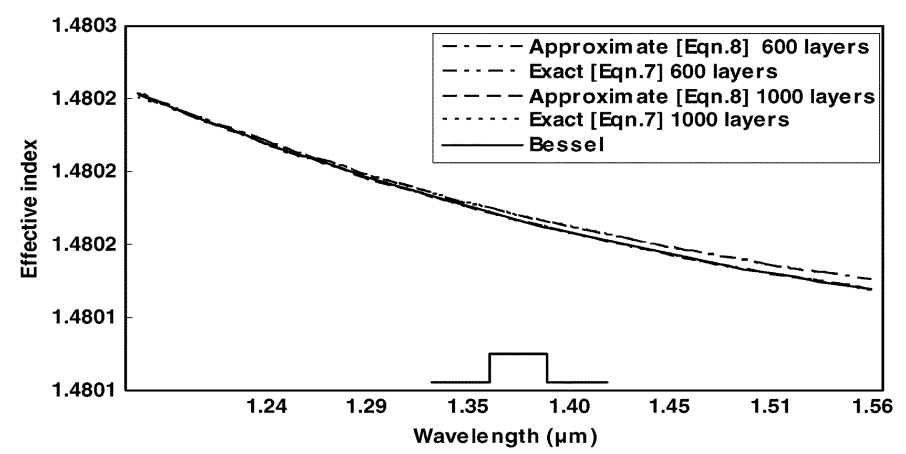

Fig. 3. Effective mode index versus wavelength curves for step index single mode optical fiber with core radius $a_{1}=2.2 \mu \mathrm{m}$ and $\Delta=0.012$.

\section{NUMERICAL RESUlTS AND DisCUSSION}

For our numerical results we consider an optical fiber with the well known refractive-index profile radial dependence as follows [13]:

$$
n(\bar{r})= \begin{cases}n_{1}\left(1-\Delta\left(\frac{\bar{r}}{a}\right)^{\alpha}\right), & \frac{\bar{r}}{a}<1 \\ n_{2}, & \frac{\bar{r}}{a}>1\end{cases}
$$

where $\Delta \equiv\left(n_{1}-n_{2}\right) / n_{1}, n_{1}$ is the maximal refractive index, $n_{2}$ is the refractive index of the outer and uniform cladding, $\alpha$ controls the decay or growth of the profile envelope, $a$ is the normalized core radius, and $\bar{r}$ is the normalized cylindrical layer radius. A variety of refractive index profiles can be generated by varying $\alpha$ ( $\alpha=1$ triangular profile, $\alpha=2$ parabolic profile, $\alpha=\infty$ step profile).

Furthermore, in order to introduce refractive index wavelength dependence, since (21) is proportional to $n$, it scales as function of wavelength according to (12).

Convergence and accuracy are important factors for any numerical technique. We have chosen the typical standard step, triangular, and linear chirp index fiber profiles since the results are well known. For all our computations, we have chosen the thickness of the cylindrical layers, inside and outside the core, to be $\delta \bar{r} / \bar{r}=0.02$, and using 600 and 1000 layers are in general sufficient for this $\delta \bar{r} / \bar{r}$ ratio.

We calculate the delay and then dispersion characteristics for step index optical fibers by applying our technique and using delay and dispersion equations, (13) and (14), within the wavelength range used in optical communications. The step index profile optical fiber used has typical values of core radius $a_{1}=$ $2.2 \mu \mathrm{m}$ and $\Delta=0.012$ [1]. In Fig. 3, we plot and compare the exact normalized propagation constant (effective mode index) for the step index fiber calculated using Bessel functions, with the results obtained with our T-L technique. We present our T-L results in two curves, one using the exact impedance equations (7) and the other plot using the approximate impedance equations (8). We also present curves by varying as a parameter the number of layers set to 600 and 1000 for our algorithm. The numerical results show $\delta \bar{r} / \bar{r}=0.02$ offers good homogeneity to the cylindrical layers we use and we obtain accurate results. With 1000 layers, the algorithm using either (7) or (8) gives us accurate results in agreement to the exact (Bessel functions) 
TABLE I

ACCuracy of the T-L Method FOR Calculating $\bar{\beta}$ FOR a SteP INDEX OPTICAL FIBER AT $\lambda_{1.3}=1.30103 \mu \mathrm{m}$

\begin{tabular}{|c|c|c|c|}
\hline & $\bar{\beta}$ at $\lambda_{1.3}$ & $\Delta \bar{\beta}=\frac{\left(\bar{\beta}-\bar{\beta}_{\text {bessel }}\right)}{\bar{\beta}_{\text {bessel }}}$ \\
\hline \multicolumn{2}{|c|}{ Bessel Function ( $\left.\bar{\beta}_{\text {bessel }}\right)$} & 1.48020635 & 0.0 \\
\hline \multirow{4}{*}{$\begin{array}{c}\text { T-L } \\
\text { Method }\end{array}$} & $\begin{array}{c}\text { Exact [Eqn.7] } \\
600 \text { layers }\end{array}$ & 1.48020794 & $1.0742 \times 10^{-4}$ \\
\hline & $\begin{array}{c}\text { Approximate } \\
\text { [Eqn.8] } \\
\text { 600 layers } \\
\end{array}$ & 1.48020795 & $1.0809 \times 10^{-4}$ \\
\hline & $\begin{array}{c}\text { Exact [Eqn.7] } \\
1000 \text { layers }\end{array}$ & 1.48020644 & $6.0802 \times 10^{-6}$ \\
\hline & $\begin{array}{c}\text { Approximate } \\
\text { [Eqn.8] } \\
\text { 1000 layers } \\
\end{array}$ & 1.48020645 & $6.7558 \times 10^{-6}$ \\
\hline
\end{tabular}

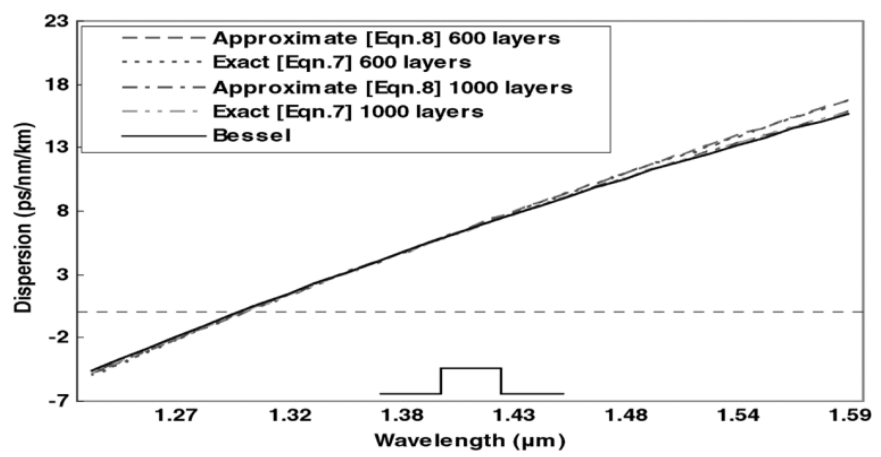

Fig. 4. Dispersion versus wavelength curves of step index optical fiber with core radius $a_{1}=2.2 \mu \mathrm{m}$ and $\Delta=0.012$.

while maintaining efficient computation speed. Therefore, we choose it for the dispersion calculation. In Table I, we demonstrate the accuracy of the T-L method in calculating the effective mode index at the wavelength $1.30103 \mu \mathrm{m}$ using (7) and (8). Both compare very well to the exact Bessel function solution. Results based on (7) are slightly closer to the result from Bessel functions. Both (7) and (8) however offer excellent accuracy. Fig. 4 compares dispersion results using our algorithm for the range 1.2-1.6 $\mu \mathrm{m}$. It includes a dispersion curve generated using Bessel functions for the effective index and dispersion plots using effective index obtained by T-L method, (7) and (8). As expected, for the step index optical fiber the zero dispersion point is at $1.30103 \mu \mathrm{m}$. Table II shows a comparison of some numerical results of calculated zero dispersion wavelengths. The results of T-L method agree very well with the result from Bessel functions. As expected, using (7) gives slightly more accurate results than (8), however, for 1000 layers this difference is not significant. The two dispersion curves in Fig. 5 are derived using Bessel functions for the exact effective mode index. One dispersion plot uses numerical differentiation and the other uses our dispersion algorithm. The zero dispersion wavelength obtained using our algorithm is $\lambda_{0}=1.30103 \mu \mathrm{m}$ and $\lambda_{0}=1.30835 \mu \mathrm{m}$ based on numerical differentiation, respectively. The result demonstrates the accuracy of our algorithm (exact $\lambda_{0}=1.30103 \mu \mathrm{m}$ ).

To allow further comparison with a recent publication on the dispersion calculation [7], we also make use of triangular refractive index profile fibers. Fig. 6 shows dispersion curves plotted

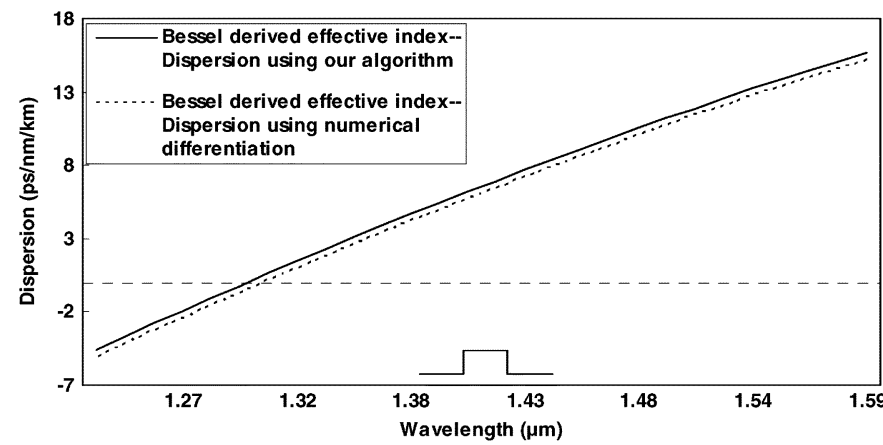

Fig. 5. Dispersion of fundamental mode of a step index optical fiber using our algorithm compared to the result using numerical differentiation for a core radius $a_{1}=2.2 \mu \mathrm{m}$ and $\Delta=0.012$.

TABLE II

ACCURACY OF THE T-L METHOD FOR CALCUlATING THE ZERO DisPERSION WAVELENGTH $\lambda_{0}$ FOR A STEP INDEX OPTICAL FIBER

\begin{tabular}{c|c|c|c}
\hline \multicolumn{1}{c|}{} & $\lambda_{0}\left(10^{-6}\right)$ & $\Delta \lambda=\frac{\left(\lambda_{0}-\lambda_{1.3}\right)}{\lambda_{1.3}}(\%)$ \\
\hline \multirow{5}{*}{ Bessel Function $\left(\lambda_{1.3}\right)$} & 1.30103 & 0.0 \\
\hline \multirow{4}{*}{ T-L } & $\begin{array}{c}\text { Exact [Eqn.7] } \\
\text { 600 layers }\end{array}$ & 1.30183 & 0.06149 \\
\cline { 2 - 4 } & $\begin{array}{c}\text { Approximate } \\
\text { [Eqn.8] } \\
\text { 600 layers }\end{array}$ & 1.30218 & 0.08839 \\
\cline { 2 - 4 } & $\begin{array}{c}\text { Exact [Eqn.7] } \\
\text { 1000 layers }\end{array}$ & 1.30133 & 0.02306 \\
\cline { 2 - 4 } & $\begin{array}{c}\text { Approximate } \\
{[\text { Eqn.8] }} \\
\text { 1000 layers }\end{array}$ & 1.30168 & 0.04996 \\
\hline
\end{tabular}

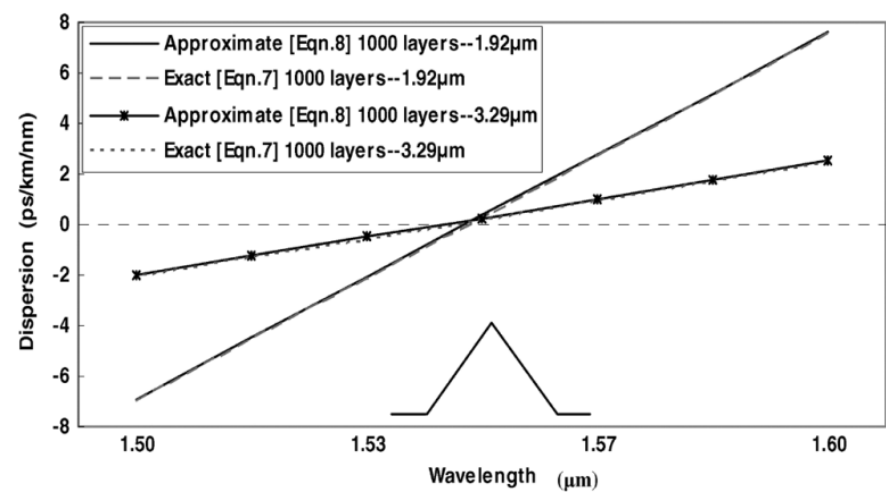

Fig. 6. Variation of dispersion versus wavelength curves for the triangular refractive index profile optical fiber with $\Delta=0.01$ at the two values of core radius which make zero dispersion point at $1.55 \mu \mathrm{m}$.

against wavelength for the two values of core radius $a_{1}$, namely, $1.92 \mu \mathrm{m}$ and $3.29 \mu \mathrm{m}$, and $\Delta=0.01$, with zero dispersion wavelengths at $1.55 \mu \mathrm{m}$. Fig. 6 shows the excellent agreement in the calculated dispersion for triangular optical fibers using the different methods with different core radii. In this case there is negligible difference between the results using (7) and (8). The results match perfectly with those given in [7].

It is well known that the dispersion flattened char acteristics of an optical fiber is very important for wavelength division multiplexing (WDM) optical systems. There have been many attempts to design dispersion flattened optical fibers [12], [14]. 


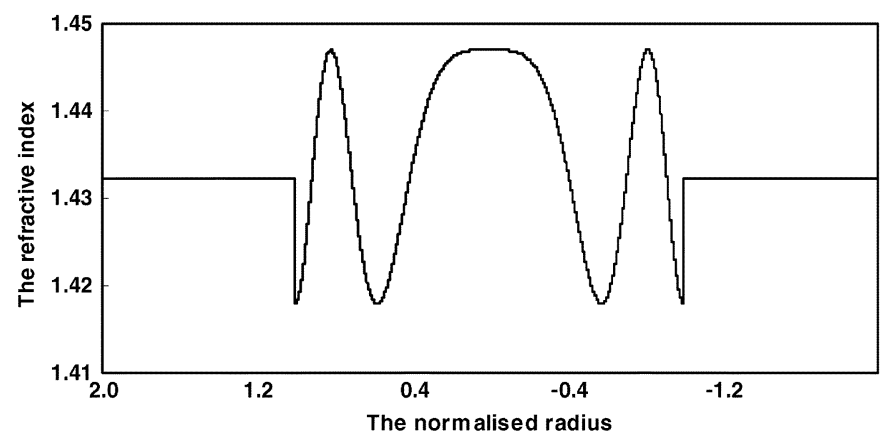

Fig. 7. Linear chirp refractive index profile of core radius $a_{1}=7.2 \mu \mathrm{m}$ and $\Delta=0.0102$.

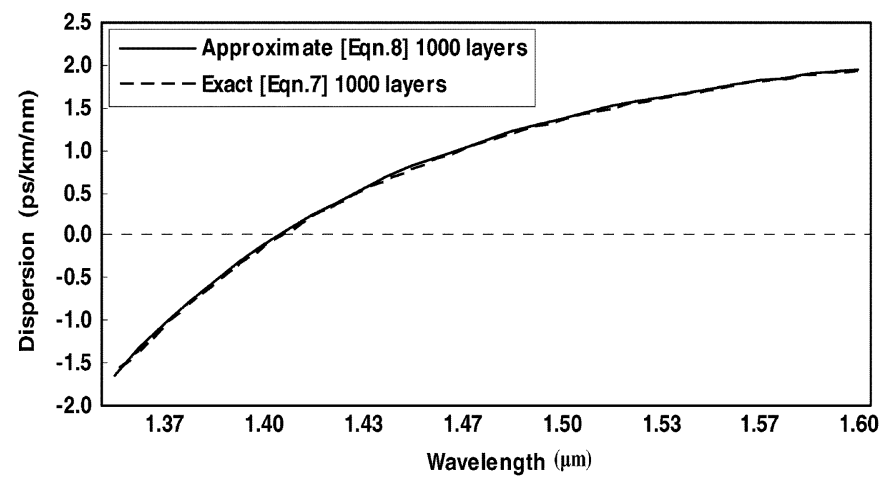

Fig. 8. Dispersion versus wavelength curves for the linear chirp refractive index profile of Fig. 7 over a wavelength range from 1.35 to $1.6 \mu \mathrm{m}$.

We demonstrate that our T-L circuit method can be used in the design of dispersion flattened optical fibers quite efficiently. We consider the optical fiber with linear chirp refractive index profile, of core radius $a_{1}=7.2 \mu \mathrm{m}$ and $\Delta=0.0102$, as shown in Fig. 7, and studied in [12]. Fig. 8 shows the calculated dispersion as a function of wavelength using our method. It can be seen that the dispersion magnitude is less than $2 \mathrm{ps} / \mathrm{nm} / \mathrm{km}$ over the entire range of $1.35-1.6 \mu \mathrm{m}$ wavelength. There is no significant difference using the T-L method with (7) or (8). The results in Fig. 8 also agree very well with the results in [12].

\section{CONCLUSION}

In this paper, a new, efficient, and accurate algorithm for calculating the mode dispersion of cylindrical dielectric waveguides has been developed from first principles. This method uses T-L representation of cylindrical dielectric waveguides and relies on the modeling of a thin uniform concentric cylindrical layer of an optical fiber to a T-L circuit. The method requires knowledge of only the mode propagation constant and the refractive index profile. It is direct and exact, and avoids the use of numerical differentiation twice. It may be especially useful for designing and predicting complex refractive index profile optical fibers where the earlier reported approximate methods are quite slow. We have demonstrated the performance of this technique by evaluating dispersion versus wavelength for step, triangular and linear chirp index profile optical fibers. The results support the claim that this algorithm provides direct calculation of dispersion with very good accuracy.

\section{APPENDIX A}

To shorten the equations, we make the following definitions:

$$
\begin{aligned}
& A=\beta^{2}+\left(\frac{l}{r}\right)^{2}, \quad B=\sinh (\gamma \delta r), \quad C=\cosh (\gamma \delta r), \\
& D=\cosh \left(\gamma \frac{\delta r}{2}\right), \quad E=\tanh \left(\gamma \frac{\delta r}{2}\right) .
\end{aligned}
$$

The first derivatives of the exact impedance equations (7)

$$
\left.\begin{array}{rl}
\frac{\partial Z_{P}}{\partial \beta} & =\frac{Z_{0}}{n r k_{0}}\left(\frac{\frac{\partial \gamma}{\partial \beta}}{A B}-\gamma\left(\frac{2 \beta}{A^{2} B}+\frac{C \delta r \frac{\partial \gamma}{\partial \beta}}{A B^{2}}\right)\right) \\
\frac{\partial Z_{P}}{\partial \lambda} & \left.=\frac{Z_{0}}{r k_{0} A} \frac{\frac{\partial \gamma}{\partial \lambda}(n B)-\gamma\left(\frac{\partial n}{\partial \lambda} B+n C \delta r \frac{\partial \gamma}{\partial \lambda}\right)}{n^{2} B^{2}}\right\} \\
\frac{\partial Z_{B}}{\partial \beta} & =\left(C \delta r \frac{\partial \gamma}{\partial \beta} E+\frac{B}{D^{2}} \frac{\delta r}{2} \frac{\partial \gamma}{\partial \beta}\right) Z_{P}+B E \frac{\partial Z_{P}}{\partial \beta} \\
\frac{\partial Z_{B}}{\partial \lambda} & =\left(C \delta r \frac{\partial \gamma}{\partial \lambda} E+\frac{B}{D^{2}} \frac{\delta r}{2} \frac{\partial \gamma}{\partial \lambda}\right) Z_{P}+B E \frac{\partial Z_{P}}{\partial \lambda}
\end{array}\right\}
$$

where

$$
\begin{aligned}
& \frac{\partial \gamma}{\partial \beta}=\frac{\partial}{\partial \beta}\left(\sqrt{\gamma^{2}}\right)=\frac{1}{2} \frac{2 \beta-\frac{2 n l k_{0}\left(l^{2}-\beta^{2} r^{2}\right)}{A^{2} r^{4}}}{\sqrt{A-n^{2} k_{0}^{2}-\frac{2 n \beta l k_{0}}{A r^{2}}}} \\
& \frac{\partial \gamma}{\partial \lambda}=\frac{1}{2} \frac{-\frac{\partial n}{\partial \lambda} k_{0}\left(2 n k_{0}+\frac{2 \beta l}{A r^{2}}\right)}{\sqrt{A-n^{2} k_{0}^{2}-\frac{2 n \beta l k_{0}}{A r^{2}}}} .
\end{aligned}
$$

The second derivatives of the exact impedance equations (7)

$$
\begin{aligned}
\frac{\partial^{2} Z_{P}}{\partial \beta \partial \lambda}= & -\frac{\frac{\partial n}{\partial \lambda}}{n^{2}} \frac{Z_{0}}{r k_{0}}\left(\frac{\frac{\partial \gamma}{\partial \beta}}{A B}-\gamma\left(\frac{2 \beta}{A^{2} B}+\frac{C \delta r \frac{\partial \gamma}{\partial \beta}}{A B^{2}}\right)\right) \\
& +\frac{Z_{0}}{n r k_{0}}\left(\frac{1}{A} \frac{\frac{\partial^{2} \gamma}{\partial \beta \partial \lambda} B-\frac{\partial \gamma}{\partial \beta} C \delta r \frac{\partial \gamma}{\partial \lambda}}{\sinh ^{2}\left(\gamma \frac{\delta \bar{r}}{k_{0}}\right)}-\frac{\partial \gamma}{\partial \lambda}\right. \\
& \times\left(\frac{2 \beta}{A^{2} B}+\frac{C \delta r \frac{\partial \gamma}{\partial \beta}}{A B^{2}}\right)-\gamma\left(\frac{2 \beta}{A^{2}} \frac{-C \delta r \frac{\partial \gamma}{\partial \lambda}}{B^{2}}+\frac{\delta r}{A}\right. \\
& \left.\left.\times \frac{\left(B \delta r \frac{\partial \gamma}{\partial \lambda} \frac{\partial \gamma}{\partial \beta}+C \frac{\partial^{2} \gamma}{\partial \beta \partial \lambda}\right) B^{2}-2 C^{2} \frac{\partial \gamma}{\partial \lambda} \frac{\partial \gamma}{\partial \beta} B \delta r}{B^{4}}\right)\right)
\end{aligned}
$$

$$
\begin{aligned}
\frac{\partial^{2} Z_{P}}{\partial \lambda^{2}}= & \frac{Z_{0}}{r k_{0} A} \frac{1}{n^{4} B^{4}}\left(\frac{\partial^{2} \gamma}{\partial \lambda^{2}} n B-\gamma\right. \\
& \times\left(\frac{\partial^{2} n}{\partial \lambda^{2}} B+2 \frac{\partial n}{\partial \lambda} C \delta r \frac{\partial \gamma}{\partial \lambda}\right. \\
& \left.\left.+n(\delta r)^{2}\left(\frac{\partial \gamma}{\partial \lambda}\right)^{2} B+n \delta r C \frac{\partial^{2} \gamma}{\partial \lambda^{2}}\right)\right) n^{2} B^{2} \\
& -\left(\frac{\partial \gamma}{\partial \lambda} n B-\gamma\left(\frac{\partial n}{\partial \lambda} B+n C \delta r \frac{\partial \gamma}{\partial \lambda}\right)\right) \\
& \times\left(2 n \frac{\partial n}{\partial \lambda} B^{2}+2 n^{2} B C \delta r \frac{\partial \gamma}{\partial \lambda}\right)
\end{aligned}
$$

$\frac{\partial^{2} Z_{B}}{\partial \beta \partial \lambda}=\left(\delta r B \delta r \frac{\partial \gamma}{\partial \lambda} \frac{\partial \gamma}{\partial \beta} E\right.$ 


$$
\begin{aligned}
& \left.+C\left(\frac{\partial^{2} \gamma}{\partial \beta \partial \lambda} E+\frac{\partial \gamma}{\partial \lambda} \frac{\partial \gamma}{\partial \beta} \frac{\delta r}{2} \frac{1}{D^{2}}\right)\right) \\
& +\frac{\delta r}{2}\left(\left(C \delta r \frac{\partial \gamma}{\partial \lambda} \frac{\partial \gamma}{\partial \beta}+B \frac{\partial^{2} \gamma}{\partial \beta \partial \lambda}\right) D^{2}\right. \\
& \left.\left.-B \frac{\partial \gamma}{\partial \beta} \delta r D \sinh \left(\gamma \frac{\delta r}{2}\right) \frac{\partial \gamma}{\partial \lambda}\right)\right) \frac{Z_{P}}{D^{4}} \\
& +\left(C \delta r \frac{\partial \gamma}{\partial \beta} E+B \frac{\partial \gamma}{\partial \beta} \frac{\delta r}{2} \frac{1}{D^{2}}\right) \frac{\partial Z_{P}}{\partial \lambda} \\
& +\left(C \delta r \frac{\partial \gamma}{\partial \lambda} E+B \frac{\partial \gamma}{\partial \lambda} \frac{\delta r}{2} \frac{1}{D^{2}}\right) \frac{\partial Z_{P}}{\partial \beta}+B E \frac{\partial^{2} Z_{P}}{\partial \beta \partial \lambda}
\end{aligned}
$$

$$
\begin{aligned}
\frac{\partial^{2} Z_{B}}{\partial \lambda^{2}}= & \left(\delta r \left(B \delta r\left(\frac{\partial \gamma}{\partial \lambda}\right)^{2} E\right.\right. \\
& \left.+c C\left(\frac{\partial^{2} \gamma}{\partial \lambda^{2}} E+\left(\frac{\partial \gamma}{\partial \lambda}\right)^{2} \frac{\delta r}{2} \frac{1}{D^{2}}\right)\right) \\
& +\frac{\delta r}{2}\left(\left(C \delta r\left(\frac{\partial \gamma}{\partial \lambda}\right)^{2}+B \frac{\partial^{2} \gamma}{\partial \lambda^{2}}\right) D^{2}\right. \\
& \left.\left.-B \frac{\partial \gamma}{\partial \lambda} D \sinh \left(\gamma \frac{\delta r}{2}\right) \frac{\partial \gamma}{\partial \lambda} \delta r\right)\right) \frac{Z_{P}}{D^{4}} \\
& +2\left(C \delta r \frac{\partial \gamma}{\partial \lambda} E+B \frac{\partial \gamma}{\partial \lambda} \frac{\delta r}{2} \frac{1}{D^{2}}\right) \frac{\partial Z_{P}}{\partial \lambda}+B E \frac{\partial^{2} Z_{P}}{\partial \lambda^{2}}
\end{aligned}
$$

where

$$
\begin{aligned}
\frac{\partial^{2} \gamma}{\partial \beta \partial \lambda}= & \left(A-n^{2} k_{0}^{2}-\frac{2 n \beta l k_{0}}{A r^{2}}\right)^{-\frac{3}{2}}\left(k_{0}^{2} n \frac{\partial n}{\partial \lambda}+\frac{\beta l k_{0}}{A r^{2}} \frac{\partial n}{\partial \lambda}\right) \\
& \times\left(\beta-\frac{n l k_{0}\left(l^{2}-\beta^{2} r^{2}\right)}{A^{2} r^{4}}\right) \\
& +\left(A-n^{2} k_{0}^{2}-\frac{2 n \beta l k_{0}}{A}\right)^{-\frac{1}{2}} \frac{l k_{0}\left(\beta^{2} r^{2}-l^{2}\right)}{A^{2} r^{4}} \frac{\partial n}{\partial \lambda} \\
\frac{\partial^{2} \gamma}{\partial \lambda^{2}}= & \frac{1}{2}\left(\frac{1}{2}\left(A-n^{2} k_{0}^{2}-\frac{2 n \beta l k_{0}}{A}\right)^{-\frac{3}{2}}\right. \\
& \times\left(\frac{\partial n}{\partial \lambda} k_{0}\left(2 n k_{0}+\frac{2 \beta l}{A}\right)\right)^{2} \\
& -\left(A-n^{2} k_{0}^{2}-\frac{2 n \beta l k_{0}}{A}\right)^{-\frac{1}{2}} \\
& \left.\times\left(2 k_{0}\left(\left(\frac{\partial n}{\partial \lambda}\right)^{2}+n \frac{\partial^{2} n}{\partial \lambda^{2}}\right)+\frac{2 \beta l k_{0} \frac{\partial^{2} n}{\partial \lambda^{2}}}{A}\right)\right) .
\end{aligned}
$$

\section{REFERENCES}

[1] B. J. Ainslie and C. R. Day, "A review of single mode fiber with modified dispersion characteristics," J. Lightw. Technol., vol. LT-4, no. 8, pp. 967-979, Aug. 1986.

[2] R. A. Sammut, "Analysis of approximations for the mode dispersion in monomode fiber," Electron Lett., vol. 15, pp. 590-591, 1979.

[3] W. L. Mammel and L. G. Cohen, "Numerical prediction of fiber transmission as characteristics from arbitrary refractive-index profiles," Appl. Opt., vol. 21, pp. 699-703, 1982.

[4] E. K. Sharma, A. Sharma, and I. C. Goyal, "Propagation characteristics of single mode optical fibers with arbitrary index profiles: A simple numerical approach," IEEE J. Quantum Electron., vol. QE-18, no. 10, pp. 1484-1489, Oct. 1982.
[5] A. Sharma and S. Banerjee, "Chromatic dispersion in single mode fiber with arbitary index profile: A simple method for exact numerical evaluation," J. Lightw. Technol., vol. 7, no. 12, pp. 1919-1923, 1989.

[6] X. Qian and A. C. Boucouvalas, "Propagation characteristics of single-mode optical fibers with arbitrary complex index profiles," IEEE J. Quantum Electron., vol. 40, no. 6, pp. 771-777, Jun. 2004.

[7] M. Y. El-Ibiary, "Parameter optimization in graded-index dispersionshifted single-mode fibers," J. Lightw. Technol., vol. LT-4, no. 4, pp. 364-367, Mar. 1986

[8] S. P. Survaiya and R. K. Shevgaonkar, "Dispersion characteristics of an optical fiber having linear chirp refractive index profile," J. Lightw. Technol., vol. 17, no. 10, pp. 1797-1805, Oct. 1999.

[9] X. Qian and A. C. Boucouvalas, "Optical fiber refractive index profile synthesis from near field," in Proc. IEEE Globecom Conf., San Francisco, CA, 2003, pp. 2669-2673.

[10] A. C. Boucouvalas and C. D. Papageorgiou, "Cutoff frequencies in optical fibers of arbitrary refractive index profile using the 'resonance' technique," IEEE J. Quantum Electron., vol. 18, pp. 2027-2031, 1982.

[11] R. B. Dyott and J. R. Stern, "Group delay in glass fiber waveguide," in Proc. Trunk Telecommunication Guided Waves Conf., London, UK, 1970 , pp. 82-84.

[12] A. Safaai-Jazi and L. J. Lu, "Accuracy of approximate methods for the evaluation of chromatic dispersion in dispersion-flattened fibers," $J$ Lightw. Technol., vol. 8, no. 8, pp. 1145-1150, Aug. 1990.

[13] M. J. Adams, An Intriduction to Optical Waveguides. New York: Wiley, 1981

[14] P. Francois, "Zero dispersion in attenuation optimized doubly clad fibers," J. Lightw. Technol., vol. LT-1, no. 1, pp. 26-37, Mar. 1983.

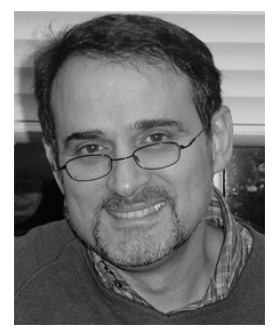

Anthony C. Boucouvalas (S'81-M'82-SM'00F'02) received the B.Sc. degree in electrical and electronic engineering from Newcastle upon Tyne University, Newcastle, U.K., in 1978, the M.Sc. and D.I.C. degrees in communications engineering from Imperial College, University of London, London, U.K., in 1979, and the Ph.D. degree in fiber optics from Imperial College, in 1982.

Subsequently, he joined the GEC Hirst Research Center and became Group Leader and Divisional Chief Scientist working on fiber-optic components, measurements, and sensors until 1987, when he joined Hewlett Packard Laboratories (HP) as a Project Manager. At HP, he worked in the areas of optical communication systems, optical networks, and instrumentation, until 1994, when he joined Bournemouth University, Bournemouth, U.K. In 1996, he became a Professor in Multimedia Communications, and in 1999 he became Director of the Microelectronics and Multimedia research Center. His current research interests span the fields of wireless communications, optical fiber communications and components, multimedia communications, and human-computer interfaces where he has published over 200 papers. He has contributed to the formation of $\operatorname{IrDA}$ as an industry standard and he is now a member of the IrDA Architectures Council contributing on new IrDA standards.

Dr. Boucouvalas is a Fellow of the Royal Society for the encouragement of Arts, Manufacturers and Commerce, Fellow of Institue of Electrical Engineers (IEE) and, in 2002, became Fellow of IEEE, "for contributions to Optical fiber components and optical wireless communications." He is a Member of the New York Academy of Sciences and ACM. He is an Editor of the IEEE Wireless Communications Magazine, IEEE TRANSACTIONS ON WIRELESS NETWORKS, Associate Editorial Member for the Wireless Communications and Mobile Computing Journal and Vice Chairman of the IEEE UK\&RI Communications Chapter. He is in the Organizing Committee of the International Symposium on Communication Systems Networks and Digital Signal Processing, (CSNDSP), and a member of Technical Committees in numerous conferences.

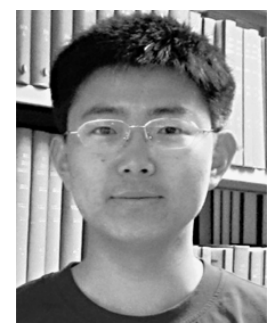

Xin Qian ( $\left.\mathrm{S}^{\prime} 03\right)$ is currently working toward the Ph.D. degree at Bournemouth University, Bournemouth, U.K. His doctoral work focuses on design of optical fibers using inverse transmission-line technique.

His research interests include fiber-based optical devices, integrated optics, and high-performance optical communication systems.

Mr. Qian is a Student Member of the Optical Society of America. 